Revista Brasil. Bot., V.32, n.1, p..23-31, jan.-mar. 2009

\title{
Diatomáceas sobre Limnoperna fortunei (Dunker), molusco introduzido no Lago Guaíba, Sul do Brasil
}

\author{
LEZILDA CARVALHO TORGAN ${ }^{1,2}$, SAIONARA ELIANE SALOMONI ${ }^{1} \mathrm{e}$ \\ ALINE BRUGALLI BICCA ${ }^{1}$
}

(recebido: 10 de maio de 2007; aceito: 07 de novembro de 2008)

\begin{abstract}
Diatoms on Limnoperna fortunei (Dunker), an introduced mollusk in Guaíba Lake, southern Brazil). The taxonomic composition of diatoms present on the mollusk Limnoperna fortunei (golden mussel), introduced into Guaíba Lake through ballast water, from ships coming from Argentina, was analyzed with the intention of identifying and evaluating the occurrence of the species in relation to habitat and origin. Mollusks were obtained from a floating wooden platform and on floats situated in the northern region of Guaíba Lake, during the period of August to November 2005. The diatoms were scraped from the shells, analysed under microscope and were oxidized for identification. Results have shown that the molluskan shell may be a favorable habitat for diatom, considering the species richness. Diatom association did not demonstrate to be restricted this habitat, since the most frequent species: Cocconeis fluviatilis Wall., C. placentula Ehr. var. lineata (Ehr.) Heurck, Encyonema silesiacum (Bleisch) Mann, Eunotia pseudosudetica Metz., Lange-Bert. \& Garcia-Rodr., Gomphonema parvulum (Kütz.) Kütz., Hydrosera whampoensis (Schw.) Deby, Luticola goeppertiana (Bleisch) Mann, Melosira varians Ag., Navicula symmetrica Patr., Nitzschia palea (Kütz.) Smith, Pinnularia acrosphaeria Smith, Planothidium frequentissimum (Lange-Bert.) Round \& Bukh., Pleurosira laevis (Her.) Comp. and Ulnaria ulna (Nitzsch) Comp. were found on rocky substrata and in the plankton of the Guaíba Lake hydrographic basin. Therefore, Eunotia pseudosudetica, Luticola dapalis (Freng.) Mann and Gomphonema mexicanum Grun. are taxa only reported, until now, for the Rio de la Plata, Argentina and their occurrences in Guaíba Lake support the hypothesis of a recent introduction of these species through ballast water as L. fortunei.
\end{abstract}

Key-words - diatoms epizoic, introduced species, taxonomy

RESUMO - (Diatomáceas sobre Limnoperna fortunei (Dunker), molusco introduzido no Lago Guaíba, Sul do Brasil). A composição taxonômica de diatomáceas presentes sobre o molusco Limnoperna fortunei (mexilhão dourado), introduzido no Lago Guaíba através da água de lastro de navios oriundos da Argentina, foi analisada com o objetivo de identificar e avaliar a ocorrência das espécies quanto ao habitat e origem. Os moluscos foram retirados de plataforma flutuante de madeira e de bóias situadas na região norte do Lago Guaíba, no período de agosto a novembro de 2005. As diatomáceas presentes sobre as conchas dos moluscos foram raspadas, analisadas sob microscópio e posteriormente oxidadas para identificação. Os resultados demonstraram que a concha do molusco pode ser considerada um habitat favorável às diatomáceas, tendo em vista a riqueza de espécies. A associação de diatomáceas não demonstrou estar restrita a este habitat, pois as espécies mais frequentes Cocconeis fluviatilis Wall., C. placentula Ehr. var. lineata (Ehr.) Heurck, Encyonema silesiacum (Bleisch) Mann, Eunotia pseudosudetica Metz., Lange-Bert. \& Garcia-Rodr., Gomphonema parvulum (Kütz.) Kütz., Hydrosera whampoensis (Schw.) Deby, Luticola goeppertiana (Bleisch) Mann, Melosira varians Ag., Navicula symmetrica Patr., Nitzschia palea (Kütz.) Smith, Pinnularia acrosphaeria Smith, Planothidium frequentissimum (Lange-Bert.) Round \& Bukh., Pleurosira laevis (Her.) Comp. e Ulnaria ulna (Nitzsch.) Comp. são também encontradas em substratos rochosos e no plâncton na Bacia Hidrográfica do Lago Guaíba. Entretanto, Eunotia pseudosudetica, Luticola dapalis (Freng.) Mann e Gomphonema mexicanum Grun. são táxons só registrados, até o momento, para o Rio da Prata, Argentina. Estes primeiros registros nas águas do Lago Guaíba suportam a hipótese de uma recente introdução dessas espécies via água de lastro, assim como L. fortunei.

Palavras-chave - diatomáceas epizóicas, espécies introduzidas, taxonomia

\section{Introdução}

Diatomáceas epizóicas sobre cetáceos (Hustedt 1952, Holmes 1985, Holmes et al. 1993, Holmes \&

1. Fundação Zoobotânica do Rio Grande do Sul, Museu de Ciências Naturais, Rua Dr. Salvador França, 1427, 90690-000 Porto Alegre, RS, Brasil.

2._Autor para correspondência: lezilda.torgan@fzb.rs.gov.br
Nagasawa 1995, Denys 1997), aves aquáticas marinhas (Croll \& Holmes 1982), larvas de insetos (Bourrelly 1974) ou sobre microcrustáceos - copépodos e cladóceros (Ikeda 1977, Russel \& Norris 1971, Gibson 1979, Winemiller \& Winsborough 1990, Gaiser \& Bachmann 1993, 1994) têm sido objeto de diversas pesquisas. Estas investigações têm revelado novos gêneros e espécies, a presença de uma flora endêmica ou de uma flora casual, sem indicação de especificidade 
de habitat, bem como de casos de simbiose. Investigações que tratam de diatomáceas sobre moluscos em águas continentais são raras, tendo-se conhecimento somente das efetuadas por Bourrelly (1974) em que registra a presença de Cocconeis placentula Ehr. sobre pequenos gastrópodos em um arroio de confluência com o Rio Isère, França e por Rossi \& Gaillard (1979), na qual encontraram Hyalodiscus sp. sobre conchas de Littoridina, afetando o tamanho e a reprodução deste gastrópodo, no canal de irrigação do Rio Colorado, na Província de Buenos Aires, Argentina.

Considerando que as diatomáceas epizóicas fornecem informações sobre o movimento e a distribuição geográfica de seu hospedeiro, este por sua vez também pode dar indícios sobre a origem e o destino das algas a ele associadas.

O molusco bivalve Limnoperna fortunei (Dunker), conhecido vulgarmente como mexilhão dourado, foi introduzido no Lago Guaíba em 1999 (Mansur et al. 1999, 2003) através da água de lastro de navios oriundos do Sudeste Asiático, passando pelo porto de Buenos Aires, Argentina (Pastorino et al. 1993, Darrigran \& Ezcurra de Drago 2000). A falta de competidores e predadores fez com que o molusco rapidamente disseminasse e colonizasse tanto substratos naturais (juncos, rochas), quanto artificiais, tais como bóias, tubulações e cascos de navios.

A invasão de espécies exóticas via água de lastro tem sido um mecanismo eficiente na dispersão de organismos marinhos e de águas continentais. Este mecanismo, dependendo da espécie introduzida, pode contribuir para o aumento ou a diminuição da biodiversidade.

A presente investigação tem como objetivos principais identificar a composição taxonômica das diatomáceas associadas às conchas de L. fortunei e avaliar a ocorrência das espécies quanto ao habitat e origem.

\section{Material e métodos}

O Lago Guaíba, localizado entre $29^{\circ} 55^{\prime}$ e $30^{\circ} 24^{\prime}$ de latitude Sul e $51^{\circ} 01^{\prime}$ e $51^{\circ} 20^{\prime}$ de longitude Oeste, possui área de $470 \mathrm{~km}^{2}$ e banha a região metropolitana de Porto Alegre, no Estado do Rio Grande do Sul. Este lago recebe, ao norte, a contribuição de quatro rios cujas áreas de drenagem somam $82.439 \mathrm{~km}^{2} \mathrm{e}$, após o percurso de $50 \mathrm{~km}$ de extensão, deságua na laguna dos Patos, sistema que se comunica no extremo sul com o Oceano Atlântico (figura 1). Os moluscos (Limnoperna fortunei) foram retirados de plataforma flutuante de madeira (estação 1) e de bóias situadas em frente à Ponta da Cadeia (estação 2), ao bairro Menino Deus (estação 3) e ao Clube Internacional (estação 4), na região norte do Lago Guaíba, no período de agosto a novembro de 2005. As

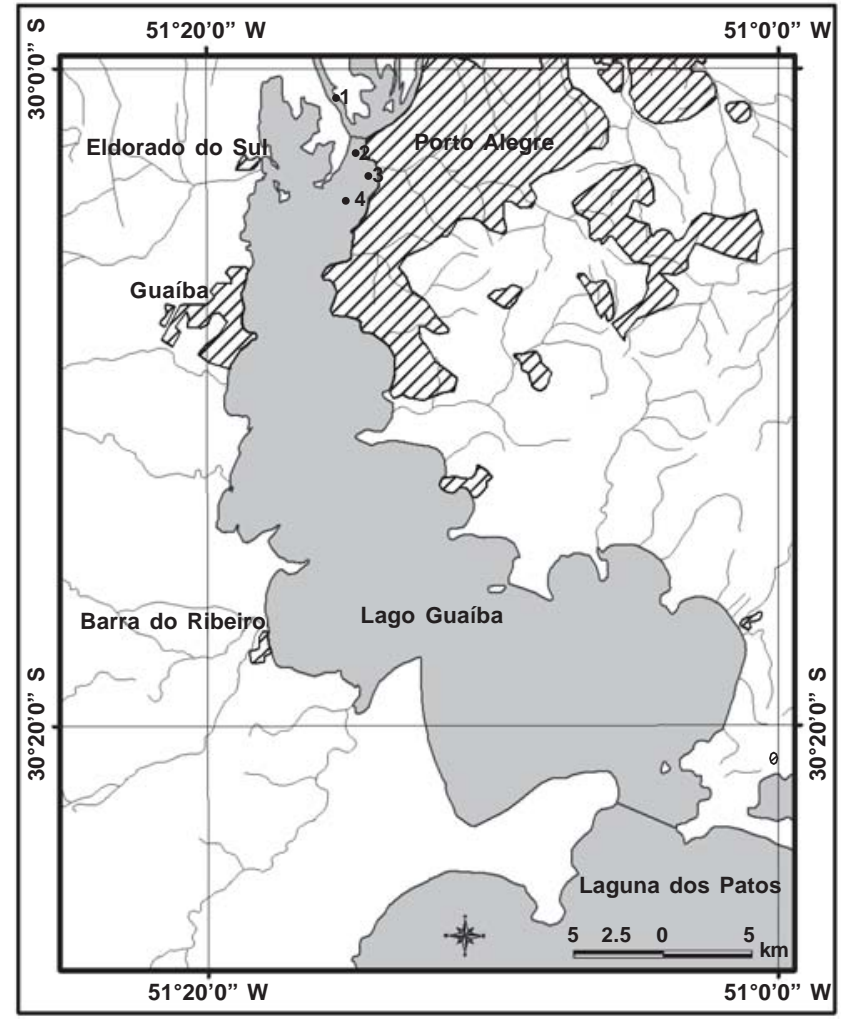

Figura 1. Mapa de localização das estações de amostragem no Lago Guaíba, Porto Alegre, Rio Grande do Sul. $(\bullet=$ estações).

Figure 1. Map of the sampling stations in the Guaíba Lake, Porto Alegre, Rio Grande do Sul. $(\bullet=$ stations $)$.

diatomáceas presentes sobre as conchas dos moluscos foram raspadas e analisadas em câmaras de sedimentação ao microscópio invertido Olympus IX 70, para observação da viabilidade das células. Um total de 40 indivíduos de tamanho médio (20 mm de comprimento) foi amostrado. Posteriormente, o material foi oxidado com água oxigenada e dicromato de potássio, lavado várias vezes com água destilada e montado em lâminas permanentes com Naphrax para análise sob microscópio Zeiss Axioplan com contraste de fase. A identificação dos táxons foi baseada, sempre que possível, na literatura original referida no texto. $\mathrm{O}$ enquadramento dos táxons seguiu o sistema de Round et al. (1990). Os táxons estão acompanhados pela variação métrica, ilustração do material examinado e comentários, quando necessário.

\section{Resultados}

Um total de 18 táxons se destacou pela frequiência sobre as conchas de Limnoperna fortunei no Lago Guaíba. Estes táxons estão distribuídos em oito ordens e 13 famílias, mostrados a seguir. 


\section{MELOSIRALES}

MELOSIRACEAE

Melosira varians Ag., Bot. Zeit., p. 628. 1827.

Variação métrica: 15,4-20 $\mu \mathrm{m}$ de diâmetro

Material examinado: BRASIL. RIo GRANDE DO SUL:

Porto Alegre, Lago Guaíba, estação 1, 10-VIII-2005, S.E.

Salomoni s.n. (HAS106612); estação 4, 26-VIII-2005,

S.E. Salomoni s.n. (HAS106630).

\section{TRICERATIALES}

TRICERATIACEA

Pleurosira laevis (Ehr.) Comp., Bacillaria, 5:177-178,

fig. 1-17, 20, 39. 1982.

Figura 2

Variação métrica: 44-59 $\mu \mathrm{m}$ de diâmetro.

Material examinado: BRASIL. Rio GRANDE Do SUL:

Porto Alegre, Lago Guaíba, estação 1, 10-VIII-2005,

S.E. Salomoni s.n. (HAS106612, lâmina 5909).

\section{BIDDULPHIALES}

\section{BIDDULPHIACEAE}

Hydrosera whampoensis (Schw.) Deby, J. Micr., 15:210, pl. 15. 1891.

Figura 3

Variação métrica: 105-132,5 $\mu$ m de distância entre os pólos.

Material examinado: BRASIL. RIo GRANDE DO SUL: Porto Alegre, Lago Guaíba, estação 2, 14-XI-2005, S.E. Salomoni s.n. (HAS106612, lâminas 5917-5918).

\section{FRAGILARIALES}

\section{FRAGILARIACEAE}

Ulnaria ulna (Nitzsch) Comp., in Jahn et al., Stud. Diatoms, p. 100. 2001.

Figura 4

Variação métrica: 101-123 $\mu \mathrm{m}$ de comprimento; $8,4 \mu \mathrm{m}$ de largura; 10 estrias em $10 \mu \mathrm{m}$.

Material examinado: BRASIL. Rio GRANDE Do Sul: Porto Alegre, Lago Guaíba, estação 2, 10-VIII-2005, S.E. Salomoni s.n. (HAS106611, lâmina 5908), estação 3, 08-IX-2005, S.E. Salomoni s.n. (HAS106641, lâmina 5914).

\section{EUNOTIALES}

\section{EUNOTIACEAE}

Eunotia pseudosudetica Metz., Lange-Bert. \& GarcíaRodr., Iconogr. Diatomol., v. 15, p. 57, pl. 24, fig. 15-18. 2005.
Figura 5

Variação métrica: 31,2-37 $\mu \mathrm{m}$ de comprimento; 5,66,0 $\mu \mathrm{m}$ de largura; 10 estrias em $10 \mu \mathrm{m}$.

Material examinado: BRASIL. Rio GRANDE do SuL: Porto Alegre, Lago Guaíba, estação 4, 26-VIII-2005, S.E. Salomoni s.n. (HAS106630, lâmina 5911).

\section{CYMBELLALES}

CYMBELLACEAE

Encyonema silesiacum (Bleisch) Mann in Round et al., The Diatoms: biology and morphology of the genera, p. 667. 1990.

Figura 6

Variação métrica: 31,2-38 $\mu \mathrm{m}$ de comprimento; 8,4$10 \mu \mathrm{m}$ de largura; 10 estrias em $10 \mu \mathrm{m}$.

Material examinado: BRASIL. Rio GRANDE DO SUL: Porto Alegre, Lago Guaíba, estação 2, 10-VIII-2005, S.E. Salomoni s.n. (HAS106611, lâmina 5907), estação 3, 08-IX-2005, S.E. Salomoni s.n. (HAS106641).

\section{GOMPHONEMATACEAE}

Gomphonema mexicanum Grun., Syn. Diat. Belgique, Atlas, pl. 24, fig. 3. 1880-1881.

Figura 7

Variação métrica: 31-32 $\mu \mathrm{m}$ de comprimento; 10 10,4 $\mu \mathrm{m}$ de largura; 10 estrias em $10 \mu \mathrm{m}$.

Material examinado: BRASIL. Rio GRANDE do Sul, Porto Alegre, Lago Guaíba, estação 1, 10-VIII-2005, S.E. Salomoni s.n. (HAS 106612, lâmina 5909, lâmina 5909).

Gomphonema parvulum (Kütz.) Kütz., Sp. Alg., p. 65. 1849.

Figura 8

Variação métrica: 22-22,4 $\mu \mathrm{m}$ de comprimento; 6,4$7 \mu \mathrm{m}$ de largura; 13-14 estrias em $10 \mu \mathrm{m}$.

Material examinado: BRASIL. Rio GRANDE DO SUL: Porto Alegre, Lago Guaíba, estação 1, 10-VIII-2005, S.E. Salomoni s.n. (HAS106612), estação 2, 10-VIII2005, S.E. Salomoni s.n. (HAS106612), estação 3, 08IX-2005, S.E. Salomoni s.n. (HAS106641, lâminas 5914 e 5915).

\section{COCCONEIDACEAE}

Cocconeis fluviatilis Wall., Notul. Nat., 331:2. 1960.

Figura 9

Variação métrica: 21-31 $\mu \mathrm{m}$ de comprimento; 13$18 \mu \mathrm{m}$ de largura; 10-12 estrias em $10 \mu \mathrm{m}$. 

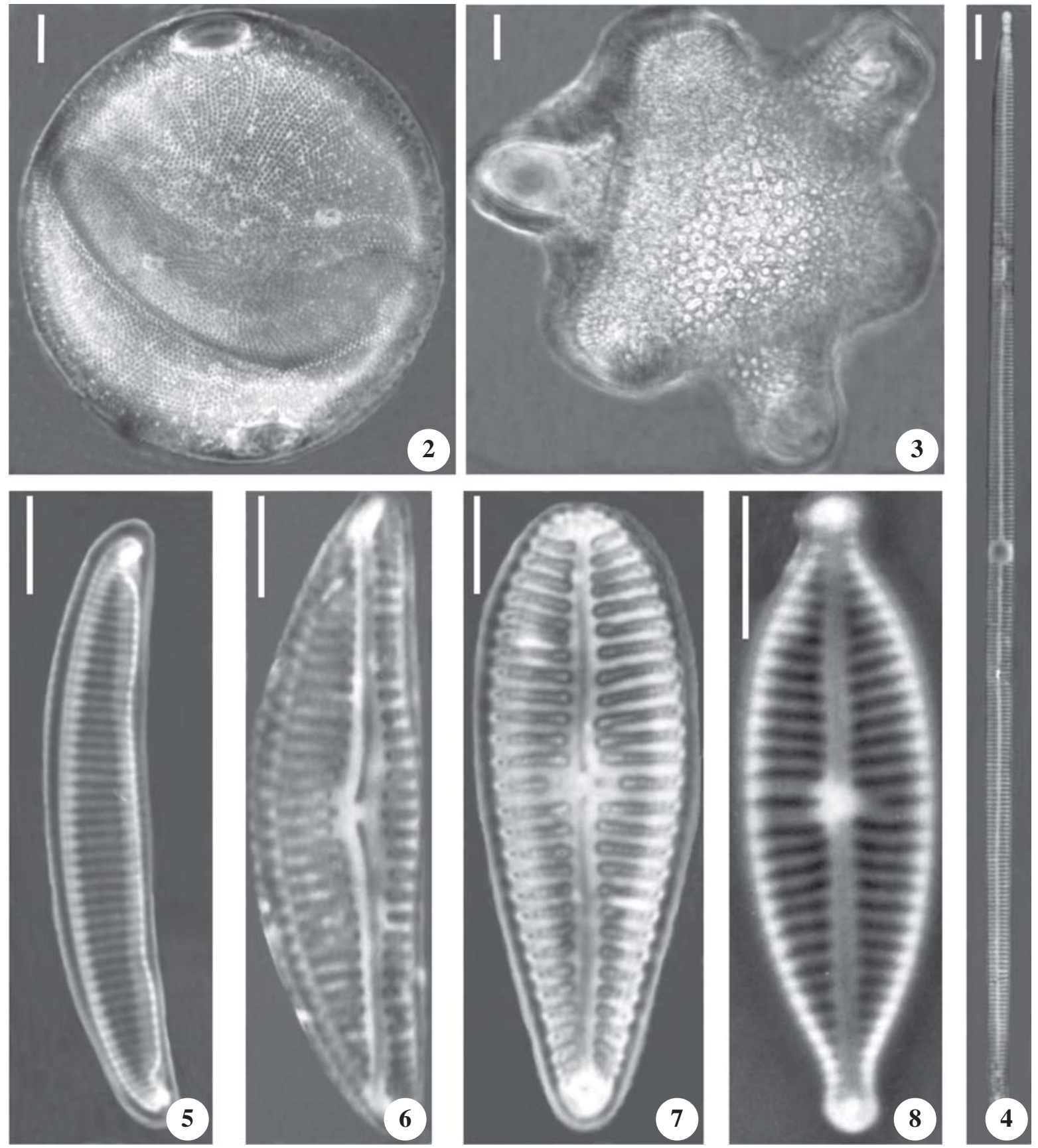

Figuras 2-8. 2. Pleurosira laevis. 3. Hydrosera whampoensis. 4. Ulnaria ulna. 5. Eunotia pseudosudetica. 6. Encyonema silesiacum. 7. Gomphonema mexicanum. 8. G. parvulum. Barra $=10 \mu \mathrm{m}(3), 5 \mu \mathrm{m}(2,4-8)$.

Figures 2-8. 2. Pleurosira laevis. 3. Hydrosera whampoensis. 4. Ulnaria ulna. 5. Eunotia pseudosudetica. 6. Encyonema silesiacum. 7. Gomphonema mexicanum. 8. G. parvulum. Bar $=10 \mu \mathrm{m}(3), 5 \mu \mathrm{m}(2,4-8)$.

Material examinado: BRASIL. Rio GRANDE Do Sul: Porto Alegre, Lago Guaíba, estação 1, 10-VIII-2005, S.E. Salomoni s.n. (HAS106612, lâmina 5907), estação 2, 10-VIII-2005, S.E. Salomoni s.n. (HAS106611, lâmina 5907), estação 3, 08-IX-2005, S.E. Salomoni s.n. (HAS106641, lâminas 5914 e 5915).
Cocconeis placentula Ehr. var. lineata (Ehr.) Heurck, Syn. Diat. Belgique, p. 133. 1885.

Figuras 10-12

Variação métrica: 24-30 $\mu \mathrm{m}$ de comprimento; 11,2$15 \mu \mathrm{m}$ de largura; $18-20$ estrias em $10 \mu \mathrm{m}$. 

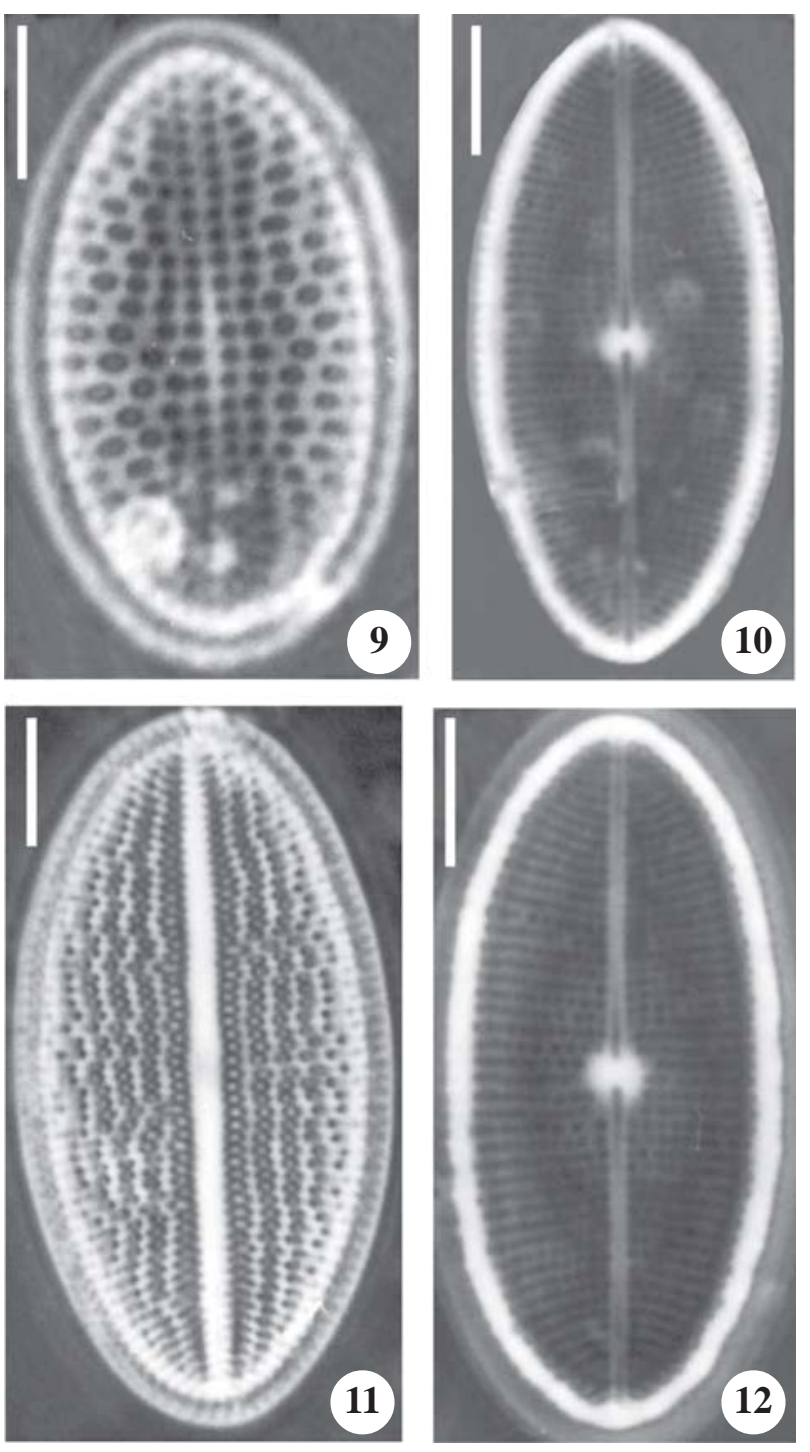

Figuras 9-12. 9. Cocconeis fluviatilis. 10-12. C. placentula var. lineata. 10,12. valva com rafe, 11. valva sem rafe. Barra $=5 \mu \mathrm{m}$.

Figures 9-12. 9. Cocconeis fluviatilis. 10-12. Cocconeis placentula var. lineata. 10, 12. valve with raphe, 11. valve without raphe. $\mathrm{Bar}=5 \mu \mathrm{m}$.

Material examinado: BRASIL. Rio GRANDE do Sul: Porto Alegre, Lago Guaíba, estação 1, 10-VIII-2005, S.E. Salomoni s.n. (HAS106612), estação 2, 10-VIII-2005, S.E. Salomoni s.n. (HAS106611, lâmina 5907), estação 4, 26-VIII-2005, S.E. Salomoni s.n. (HAS106630, lâminas 5911, 5912), estação 3, 08-IX-2005, S.E. Salomoni s.n. (HAS106641, lâmina 5914).

\section{ACHNANTHIDIACEAE}

Planothidium frequentissimum (Lange-Bert.) Round \& Bukh., Diatom Res.11(2): 352. 1996.
Figuras 13-14

Variação métrica: 20-20,8 $\mu \mathrm{m}$ de comprimento; 6-7 $\mu \mathrm{m}$ de largura; 10-14 estrias em $10 \mu \mathrm{m}$.

Material examinado: BRASIL. Rio GRANDE do SuL: Porto Alegre, Lago Guaíba, estação 4, 26-VIII-2005, S.E. Salomoni s.n. (HAS106630, lâmina 5911), estação 3, 08-IX-2005, S.E. Salomoni s.n. (HAS106641, lâmina 5914).

\section{NAVICULALES}

\section{DIADESMIDACEAE}

Luticola dapalis (Freng.) Mann, in Round et al., The Diatoms: biology and morphology of the genera, p. 670. 1990.

\section{Figura 15}

Variação métrica: $68-100,5 \mu \mathrm{m}$ de comprimento; 18,4-22,5 $\mu \mathrm{m}$ de largura; 10-12 estrias em $10 \mu \mathrm{m}$.

Material examinado: BRASIL. Rio GRANDE do SuL: Porto Alegre, Lago Guaíba, estação 1, 10-VIII-2005, S.E. Salomoni s.n. (HAS106612, lâmina 5909).

Comentários: os exemplares observados conferem com a descrição morfológica e ilustração original de Navicula dapalis (Frenguelli 1941, p. 248, est. 2, figura 14). Este autor observou somente um exemplar com 138 $\mu \mathrm{m}$ de comprimento, $38 \mu \mathrm{m}$ de largura, 7 estrias no centro e 10 estrias em $10 \mu \mathrm{m}$ nos ápices. Esta espécie foi considerada por Hustedt (1966, p. 606, fig. 1609) sinônimo de Navicula dapaloides Freng. Entretanto, considera-se que são espécies distintas e que se diferenciam, principalmente, pelo formato das valvas, pelas extremidades proximais da rafe e pela presença de estigma. Navicula dapalis possui valvas lanceoladas, levemente constrictas na região mediana, ápices subagudos, extremidades proximais da rafe inclinadas e estrias grosseiramente pontuadas, enquanto que $N$. dapaloides apresenta valvas levemente trionduladas, ápices subrostrados, extremidade proximal da rafe reta e um estigma prolongado na área central.

Luticola dapaloides (Freng.) Metz. \& Lange-Bert., Iconogr. Diatomol., 5:137. 1998.

\section{Figura 16}

Variação métrica: 58,9-94,5 $\mu \mathrm{m}$ de comprimento; 15,2-22 $\mu \mathrm{m}$ de largura; 10 estrias em $10 \mu \mathrm{m}$.

Material examinado: BRASIL. Rio GRANDE do SUL: Porto Alegre, Lago Guaíba, estação 1, 10-VIII-2005, S.E. Salomoni s.n. (HAS106612, lâmina 5909), estação 2, 10-VIII-2005, S.E. Salomoni s.n. (HAS106611, lâmina 5907). 
Comentários: os exemplares observados conferem com a descrição original de Frenguelli (1953 p.78, est. 1, fig. 20,21) diferindo apenas na largura das valvas. O material tipo originário de um arroio na Serra de Santa Vitória, a $40 \mathrm{~km}$ da catarata de Iguaçu, na Argentina, apresentou 54-144 $\mu \mathrm{m}$ de comprimento, 22-36 $\mu \mathrm{m}$ de
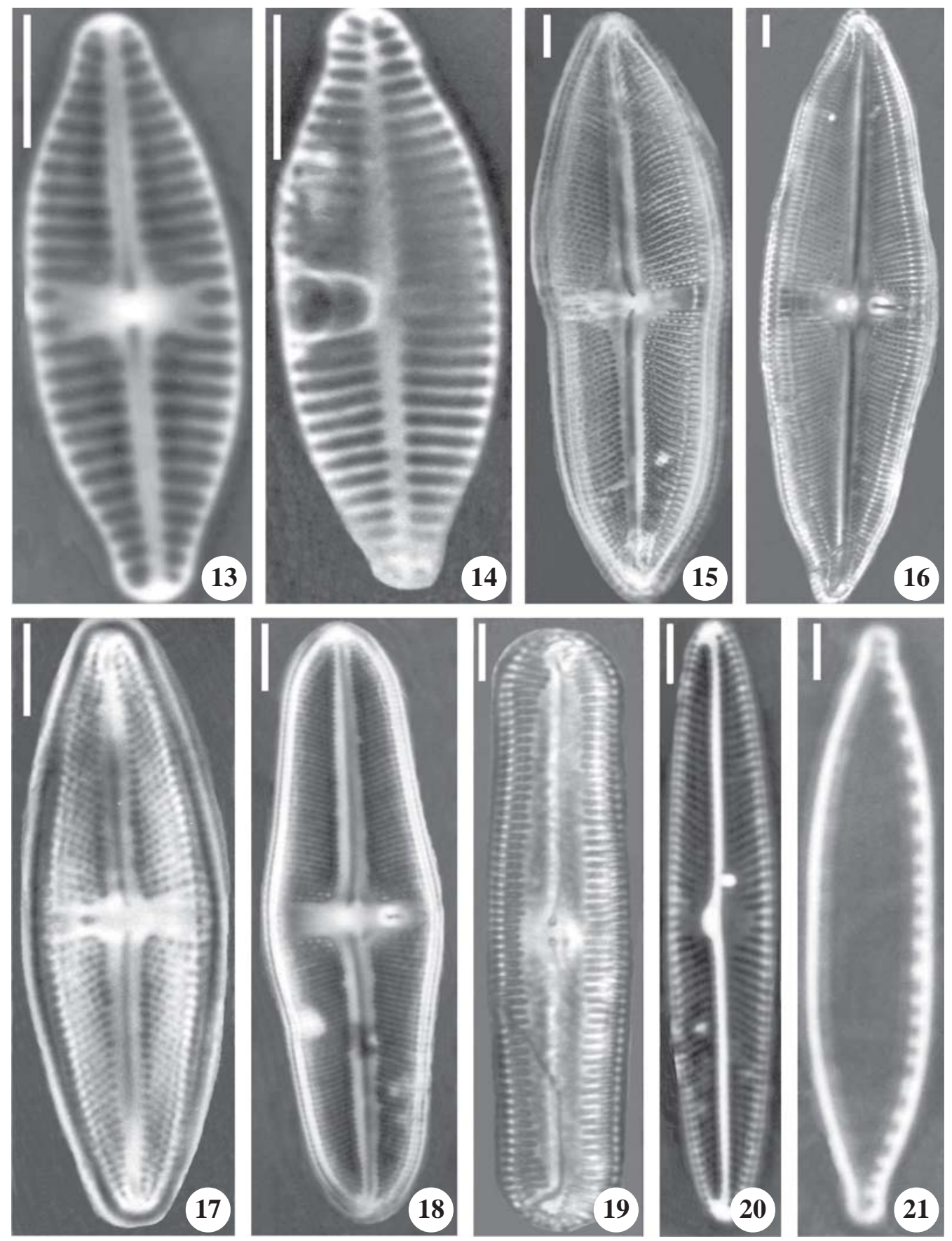

Figuras 13-21. 13, 14. Planothidium frequentissimum. 13. Valva com rafe, 14. Valva sem rafe. 15. Luticola dapalis. 16. L. dapaloides. 17. L. goeppertiana. 18. L. monita. 19. Pinnularia acrosphaeria. 20. Navicula symmetrica. 21. Nitzschia palea. Barra $=5 \mu \mathrm{m}(13-20), 2 \mu \mathrm{m}(21)$.

Figures 13-21. 13, 14. Planothidium frequentissimum. 13. Valve with raphe, 14. Valve without raphe. 15. Luticola dapalis. 16. L. dapaloides. 17. L. goeppertiana. 18. L. monita. 19. Pinnularia acrosphaeria. 20. Navicula symmetrica. 21. Nitzschia palea. Bar $=5 \mu \mathrm{m}(13-20), 2 \mu \mathrm{m}(21)$. 
largura e 10 estrias em $10 \mu \mathrm{m}$. Metzeltin \& Lange-Bertalot (1998) encontraram, em material fóssil do Quaternário na região do Amazonas, indivíduos semelhantes que denominaram de Luticola frenguellii Metz. \& LangeBert., com limites de largura e estrias mais próximos ao encontrado no Lago Guaíba. Considerando que se trata da mesma espécie, manteve-se a denominação $L$. dapaloides, por ser mais antiga.

Luticola goeppertiana (Bleisch) Mann in Round et al., The Diatoms: biology and morphology of the genera, p. 670.1990.

Figura 17

Variação métrica: 32-34 $\mu \mathrm{m}$ de comprimento; $8-9 \mu \mathrm{m}$ de largura; 14 estrias em $10 \mu \mathrm{m}$.

Material examinado: Rio Grande do Sul: Porto Alegre, Lago Guaíba, estação 3, 08-IX-2005, S.E. Salomoni s.n. (HAS 106641, lâmina 5914).

Luticola monita (Hust.) Mann, in Round et al., The Diatoms: biology and morphology of the genera, p. 671 . 1990.

Figura 18

Variação métrica: 41-44 $\mu \mathrm{m}$ de comprimento; 9,811,2 $\mu \mathrm{m}$ de largura; 21-23 estrias em $10 \mu \mathrm{m}$.

Material examinado: BRASIL. Rio GRANDE do Sul: Porto Alegre, Lago Guaíba, estação 3, 08-IX-2005, S.E. Salomoni s.n. (HAS106641, lâmina 5914).

\section{PINNULARIACEAE}

Pinnularia acrosphaeria Smith, Syn. British Diat., v.1, p. 58, pl. 19, fig. 183. 1853.

Figura 19

Variação métrica: 52-77,1 $\mu \mathrm{m}$ de comprimento; 10$11 \mu \mathrm{m}$ de largura; $12-13$ estrias em $10 \mu \mathrm{m}$.

Material examinado: BRASIL. RIo GRANDE DO SUL: Porto Alegre, Lago Guaíba, estação 3, 08-IX-2005, S.E. Salomoni s.n. (HAS106641, lâmina 5914).

\section{NAVICULACEAE}

Navicula symmetrica Patr., Bolm Mus. nac. Rio de J., 2:5, fig. 6. 1944.

Figura 20

Variação métrica: 33-49 $\mu \mathrm{m}$ de comprimento; 6,67,6 $\mu \mathrm{m}$ de largura; 12-13 estrias em $10 \mu \mathrm{m}$.

Material examinado: BRASIL. RIo GRANDE DO Sul: Porto Alegre, Lago Guaíba, estação 2, 10-VIII-2005, S.E. Salomoni s.n. (HAS106611, lâminas 5907, 5908).

\section{BACILLARIALES}

BACILLARIACEAE

Nitzschia palea (Kütz.) Smith, Syn. British Diat., v.2, p. 89. 1856.

Figura 21

Variação métrica: 21-25 $\mu \mathrm{m}$ de comprimento; 3,8$5 \mu \mathrm{m}$ de largura; 12 fíbulas em $10 \mu \mathrm{m}$.

Material examinado: BRASIL. Rio GRANDE do SuL: Porto Alegre, Lago Guaíba, estação 3, 08-IX-2005, S.E. Salomoni s.n. (HAS106641, lâmina 5914).

\section{Discussão}

A riqueza de espécies encontrada sobre as conchas de Limnoperna fortunei demonstra que este molusco pode ser considerado um habitat favorável à presença das diatomáceas. Isso se deve provavelmente por estes bivalves estarem localizados em substratos próximos à superfície da água, na zona eufótica, por disponibilizarem nutrientes inorgânicos dissolvidos no próprio meio e por servirem de suporte à fixação das diatomáceas perifíticas.

As espécies encontradas sobre o molusco possuem rafe, pela qual eliminam ao exterior mucilagem, que é composta predominantemente por polissacarídeos (Wetherbee \& Burke 1998). Esse biocomponente possibilita a aderência das diatomáceas ao substrato e esta fixação pode se dar de várias formas: pela adsorção de toda a face valvar com rafe (forma adnata), caso do gênero Cocconeis; pela formação de pedúnculos, caso de Gomphonema e Encyonema; ou pela formação de uma matriz produzida pelos primeiros colonizadores, que adere os organismos da sucessão, caso dos demais gêneros (Barber \& Haworth 1994).

A associação das espécies de diatomáceas encontradas não demonstrou estar restrita a este habitat, pois as espécies mais freqüentes Cocconeis placentula var. lineata, Gomphonema parvulum, Luticola goeppertiana, Nitzschia palea e Ulnaria ulna foram também encontradas em substratos rochosos, na Bacia Hidrográfica do Lago Guaíba (Salomoni 2006), enquanto que Cocconeis fluviatilis, Encyonema silesiacum e Hydrosera whampoensis foram registradas no plâncton por Torgan (1978), quando foi realizado o primeiro estudo florístico sobre diatomáceas neste Lago.

Entretanto, é importante mencionar o primeiro registro de ocorrência de Luticola dapalis, Eunotia pseudosudetica e Gomphonema mexicanum, espécies referidas, até o momento, somente para o Rio da Prata por Frenguelli (1941) e Metzeltin et al. (2005), 
respectivamente. Há, portanto, possibilidade destas espécies terem sido introduzidas no Lago Guaíba através da água de lastro, pois aproximadamente $45 \%$ dos navios que lastram e deslastram no porto de Porto Alegre provém da Argentina. A invasão destas espécies na região norte do Lago Guaíba, entretanto, parece não ter afetado a diversidade da flora de diatomáceas, tendo em vista a riqueza de espécies sobre Limnoperna fortunei.

Agradecimentos - À pesquisadora Maria Cristina Dreher Mansur, do Museu de Ciências e Tecnologia da Pontifícia Universidade Católica do Rio Grande do Sul (PUCRS), pelas valiosas informações prestadas, aos técnicos do Departamento Municipal de Águas e Esgotos de Porto Alegre (DMAE) pelo apoio logístico nas coletas. Ao Haywood Dail Laughinghouse IV, pela revisão do Abstract. Ao Conselho Nacional de Desenvolvimento Científico e Tecnológico $(\mathrm{CNPq})$ e à Coordenação de Ensino à Pesquisa de Ensino Superior (Capes) pela concessão de Bolsas de Pesquisa e de Pós-Graduação aos autores.

\section{Referências bibliográficas}

BARBER, H.G. \& HAWORTH, E.Y. 1994. A guide the morphology of the diatom frustule. Freshwater Biological Association Scientific Publication 44: 5-112.

BOURRELLY, P. 1974. Quelques algues fixées sur des animaux torrenticoles. Travaux Laboratoire Hydrobiologie 64-65:259-261.

CROLL, D.A. \& HOLMES, R.W. 1982. A note on the occurrence of diatoms on the feathers of diving seabirds. The Auk: A Quarterly Journal of Ornithology 99:765766.

DARRIGRAN, G. \& EZCURRA DE DRAGO, I. 2000. Invasion of the exotic freshwater mussel Limnoperna fortunei (Dunker, 1857) (Bivalvia:Mytilidae) in South America. Nautilus 114:69-73.

DENYS, L. 1997. Morphology and taxonomy of epizoic diatoms on a sperm whale. Diatom Research 12:1-18.

FRENGUELLI, J. 1941. Diatomeas del Rio de la Plata. Revista del Museo de la Plata 3:213-334.

FRENGUELLI, J. 1953. Diatomeas del Territorio Nacional de Missiones. Revista del Museo de La Ciudad Eva Peron 32:73-86.

GAISER, E.E. \& BACHMANN, R.W. 1993. The ecology and taxonomy of epizoic diatoms on cladocera. Limnology and Oceanography 38:628-637.

GAISER, E.E. \& BACHMANN, R.W. 1994. Seasonality substrate preference and attachment sites of epizoic diatoms on cladoceran zooplankton. Journal of Plankton Research 16:53-68.

GIBSON, R.A. 1979. Protoraphis atlantica sp. nov., a new marine epizoic diatom. Bacillaria 2:109-126.
HOLMES, R.W. 1985. The morphology of diatoms epizoic on cetaceans and their transfer from Cocconeis to two new genera, Bennettella and Epipellis. British Phycological Journal 20:43-57

HOLMES, R.W. \& NAGASAWA, S. 1995. Bennettella constricta (Nemoto) Holmes and Bennettella berardii sp. nov. (Bacillariophyceae; Chrysophyta) as observed on the skin of several cetacean species. Bulletin of the National Science Museum, Series B 21:29-43.

HOLMES, R.W., NAGASAWA, S. \& TAKANO, H. 1993. The morphology and geographic distribution of epidermal diatoms of the Dall's porpoise (Phocoenoides dalli True) in the Northern Pacific Ocean. Bulletin of the National Science Museum, Series B 19:1-18.

HUSTEDT, F. 1952. Diatomeen aus der Lebensgemeinschaft des Buckelwals (Megaptera nodosa Bonn.). Archiv für Hydrobiologie 46:286-298.

HUSTEDT, F. 1966. Die Kieselalgen. Deutschland, Österreichs und der Schweiz. In Kryptogamen-flora von Deutschland Österreichs und der Schweiz (L. Rabenhorst ed.). Akademische Verlagsgesellschaft, Leipzig: v.7, pt.4, p.557-816.

IKEDA, T. 1977. A pelagic marine copepod associated with diatoms. Bulletin of the Plankton Society of Japan 24:115-118.

MANSUR, M.C.D., RICHINTITI, L.M.Z. \& DOS SANTOS, C.P. 1999. Limnoperna fortunei (Dunker, 1857) molusco bivalve invasor na bacia do Guaíba, Rio Grande do Sul, Brasil. Biociências 7:147-149.

MANSUR, M.C.D., DOS SANTOS, C.P., ARRIGAN, G., HEYDRICH, I, CALLIL, C.T. \& CARDOSO, F.R. 2003. Primeiros dados quali-quantitativos do mexilhãodourado, Limnoperna fortunei (Dunker), no Delta do Jacuí, no Lago Guaíba e na laguna dos Patos, Rio Grande do Sul, Brasil e alguns aspectos de sua invasão no novo ambiente. Revista Brasileira de Zoologia 20:75-84.

METZELTIN, D. \& LANGE-BERTALOT, H. 1998. Tropical diatoms of the South America I. In Iconographia Diatomologica (H. Langue-Bertalot ed.) Koeltz Scientific Books, Koenigstein v.5.

METZELTIN, D., LANGE-BERTALOT, H. \& GARCÍARODRIGUEZ, F. 2005. Diatoms of Uruguay. In Iconographia Diatomologica (H. Langue-Bertalot ed.) Koeltz Scientific Books, Koenigstein v.15.

PASTORINO, G., DARRIGRAN, G., MARTIN, S. \& LUNASCHI, L. 1993. Limnoperna fortunei (Dunker, 1857) (Mytilidae) nuevo bivalvo invasor em aguas del Rio de la Plata. Neotropica 39:34.

ROSSI, J.B. \& GAILLARD, M.C. 1979. Epibiosis de diatomeas sobre Littoridinas (Gastropoda Hydrobiidae). Limnobios 1:333-336.

ROUND, F.E., CRAWFORD, R.M. \& MANN, D.G. 1990. The diatoms: biology and morphology of the genera. Cambridge University Press, New York. 
RUSSEL, D.J. \& NORRIS, R.E. 1971. Ecology and taxonomy of an epizoic diatom. Pacific Science 25:357-367.

SALOMONI, S.E. 2006. Epilithic diatoms as indicators of wather quality in the Gravataí River, Rio Grande do Sul, Brasil. Hydrobiologia 559:233-246.

TORGAN, L.C. 1978. Diatomáceas do "Rio" Guaíba, Porto Alegre, Rio Grande do Sul. Iheringia, Série Botânica 23:1-108.
WETHERBEE, R. \& BURKE, J.L.L. 1998. The first kiss: establishment and control of initial adhesion by raphid diatoms. Journal of Phycology 34:9-15.

WINEMILLER, K. \& WINSBOROUGH, B.M. 1990. Occurrence of epizoic communities on the parasitic copepod Lernaea crassii (Lernaeidae). The Southwestern Naturalist 35:206-210. 